\title{
Impaired face recognition does not preclude intact whole face perception
}

\author{
Romke Rouw \\ Cognitive Neuroscience Laboratory, Tilburg University, The Netherlands \\ Beatrice de Gelder
}

Cognitive Neuroscience Laboratory, Tilburg University, The Netherlands, and Neurophysiology Laboratory, Faculty of Medicine, Universite de Louvain-La-Neuve, Belgium

\begin{abstract}
We studied intact and impaired processes in a prosopagnosic patient (RP). In Experiment 1, RP showed an inversion superiority effect with both faces and objects, with better performance when stimuli were presented upside down than in normal upright orientation. In Experiment 2, we studied the effect of face configuration directly by comparing matching performance with normal vs. scrambled faces. RP was worse with normal than with scrambled faces, whereas normal controls showed an advantage of a good face context. In Experiment 3, RP showed interference from external face features on the evaluation of internal face features. These results indicate, first, that although RP is impaired in face recognition and face matching, he does still encode the whole face rather than relying completely on parts-based procedures. Second, RP has a deficit at the level of the configural processes involved in finding subtle differences between individual faces, as his performance is worse when presented with a normal face configuration than with scrambled or inverted faces.
\end{abstract}

Prosopagnosia is a rare disorder in which a patient is selectively impaired in recognizing familiar faces. Prosopagnosic patients have not lost the ability to recognize a person per se: they are still able to recognize a person by voice or a

Please address all correspondence to R. Rouw, University of Amsterdam, Department of Psychology, Roetersstraat 15, 1018 WB Amsterdam, The Netherlands .

Email: rouw@psy.uva.nl

We thank RP for his collaboration and patience and Bruno Laeng for bringing us in contact with RP. We thank Andries van der Ark for advice on statistics. We gratefully acknowledge comments from N. Donnelly and M. Craigie. We thank M. Moscovitch for providing the stimuli of the cartoon test. 
particular piece of clothing. This impairment in face recognition can extend to a problem with unfamiliar face matching as well. The specificity of the face impairment (Bodamer, 1947) has raised the intriguing question of what is "special" about recognizing faces.

One explanation of prosopagnosia is based on the notion that face recognition depends strongly on certain face specific processes, which function differently and independently from other visual recognition processes. By definition, a deficit of these processes then impairs the recognition of faces much more than the recognition of other (object) stimuli. One such proposal (Biederman \& Kalocsai, 1997; Farah, Wilson, Drain, \& Tanaka, 1998) hypothesize s that faces are represented in a relatively holistic manner (representations have little part representation), whereas object recognition is more parts based (parts are explicitly represented). In this view, impaired face recognition reflects impaired "holistic" representations, whereas objects can still be recognized by alternative (parts-based) processes.

An older proposal states that prosopagnosia is caused by a general loss of "configural processing" (Levine \& Calvanio, 1989). The ability to recognize individual features is contrasted with an inability to get an overview of sufficient features of a stimulus to allow the structuring of a coherent percept, which is specifically reflected in impaired face processing.

A different explanation of prosopagnosia is based on the assumption that the processes involved in face recognition depend on particular stimulus properties rather than on stimulus class. Thus, faces are only special in their particular combination of stimulus properties. Damasio, Damasio, and Van Hoesen (1982) advanced what Moscovitch, Winocur, and Behrmann (1997) called the "individuation hypothesis". According to this hypothesis, prosopagnosic patients are impaired at making fine discriminations necessary to see the subtle differences between exemplars of the same category. Therefore, prosopagnosics are impaired at recognizing individual faces, but can still differentiate between object classes (e.g., a chair from a table). The proper control task for individual face recognition would therefore be one requiring individual object recognition. Studies on prosopagnosic patients' performance on within-class recognition of stimuli other than faces led to mixed results. Some studies report that prosopagnosic patients did show a problem with recognition of birds (Bornstein, 1963), or cows (Bornstein, Sroka, \& Munitz, 1969). Other reports, however, show prosopagnosic patients with unimpaired recognition of sheep (McNeil \& Warrington, 1993), cows and dogs (Bruyer et al., 1983), and common objects such as chairs and glasses (Farah, Levinson, \& Klein, 1995).

In another version of the "stimulus properties" explanation there are two main factors that underlie the processes involved in face recognition, not only the factor of within-class recognition but also the expertise we hold for faces. Carey and Diamond (1994; Diamond \& Carey, 1986) distinguish first-order 
relational information (the spatial relations between parts) and second-order relational information (variations of these spatial relations relative to the common face configuration). Due to specific properties of facial stimuli, namely our expertise with faces combined with the fact that faces have a shared configuration, second-order relational information is of crucial importance in face discrimination and identification. This notion is in line with the argument (Damasio et al., 1982; Gauthier \& Tarr, 1997; Gauthier, Skudlarski, Gore, \& Anderson, 2000) that the specificity of processes involved in face recognition does not relate to the stimulus class, but rather results from the combination of subordinate (within-class) level matching task combined with a sufficient degree of expertise the observer holds for the stimuli. In this line of thought, normal observers use their expertise with the face configuration in order to achieve good performance in making the subtle discriminations between different individual faces. Furthermore, prosopagnosia occurs when this ability is somehow not available.

In this report, we did not set out to study the degree to which processes involved in face recognition are special to this stimulus class only. Rather, we examined in more detail which processes are involved in face recognition and which of these processes were spared or impaired processes in prosopagnosic patient RP. An intriguing insight on spared and impaired processes in prosopagnosia is provided by recent reports on prosopagnosic patients who show not just a loss of the normal pattern of performance, but an effect opposite from the effect found with normal controls. Farah, Wilson, Drain, and Tanaka (1995) presented prosopagnosic patient LH with upright and inverted faces. Normal controls show in this task faster and better matching of upright than inverted faces. Prosopagnosic patient LH, however, paradoxically performed better at matching inverted faces than upright faces. Farah, Wilson et al. reasoned that $\mathrm{LH}$ has intact parts-based processing with objects, whereas mandatory holistic face processes were impaired. Therefore, LH applies impaired holistic processes when presented with faces. Disturbing the face configuration (by inverting the face) provides LH with the possibility to rely on (intact) parts-based processes.

The reasoning (described above) explaining prosopagnosia as a deficit in making subtle within-class discriminations provides a different explanation for the findings by Farah, Wilson et al. LH tries to apply configural processes normally involved finding the subtle differences between normal (upright) faces as well as individual objects. These processes are particularly useful for normal controls, but LH fails. In support of this explanation, we recently studied LH (De Gelder \& Rouw, 2000b) and not only replicated Farah's finding with inverted and upright faces, but also found an inversion superiority effect in a within-category matching task with objects (shoes and houses).

As becomes clear from the descriptions provided earlier, there seems to be a general consensus on the specific importance of "configural" (or "holistic") 
information for face recognition, whereas objects can also be recognized in a parts-based or featural manner. In fact, the very good performance normally shown with recognizing or matching a face stimulus is often discussed as if it were the same issue as that of relative dependence on a certain manner of processing. But in this paper we set out to examine separately on the one hand the influence of a face configuration on matching performance and on the other hand the issue of relative dependence on "whole-based" vs. "parts-based" processes. We will first specify the terms used in this paper, and specify what these terms refer to, as different terms and definitions co-exist in the literature.

We use the terms "whole-based" vs. "parts-based" to refer to a manner of processing. Note that if a stimulus is processed as a whole rather than in terms of its parts, this still provides no information of what constitutes the "whole". Thus, "whole-based processing" (as contrasted with "parts-based processing") can mean that there is relatively little parts representation ("holistic") of the stimulus, or alternatively, that rather than information on the parts themselves, the relations between the parts ("configural") information, "first-order relational" information) are critical. To avoid confusion, we reserved the term "configuration" to refer to the presence of a specific stimulus property (namely, a normal face configuration). We will study both issues in prosopagnosic patient RP. First, we will study how the presence of a face (as compared with very similar stimuli but lacking the right configuration) influences his performance level. Second, we study whether RP's impaired face recognition performance implies that only parts-based processing is still intact.

In the first experiment, we present prosopagnosic patient RP with a face inversion task similar to that of Farah, Wilson et al. (1995), and include object stimuli as was previously done with AD (De Gelder, Bachoud-Levi, \& Degos, 1998) and with LH (De Gelder \& Rouw, 2000b). The task was also presented to normal controls. The normal controls are tested with the same task to ascertain that the expected effects can be obtained with our materials and task settings. However, we do not compare normal performance and RP's performance on each separate condition. Understandably, a patient with brain damage can show overall slower and worse performance than normal controls but we examine the pattern of performance that emerges from comparing the different conditions in one experiment.

In all experiments both accuracy and latency were measured. In many clinical studies, accuracy but not latency is reported which entails the problems that effects in response time as well as possible trade-off effects cannot be detected. Carefully chosen instructions are important to find the right balance between these two measurements. Therefore, instructions stressed both speed and accuracy and indicated that the measuring of response times still implies that $\mathrm{RP}$ (and controls) should at all times try to find the right answer. 


\section{CASE DESCRIPTION}

Patient RP is a 49-year-old man who has lost the ability to recognize faces as a consequence of a head trauma that occurred when he was seven years old. His intellectual abilities are above average and he does not suffer from any known visual dysfunction. As is to be expected in cases caused by closed head injury (see for example CK; Moscovitch et al., 1997) an MRI scan did not yield evidence of brain damage (see De Gelder \& Kanwisher, 1999). As can be seen in Appendix I, RP's problem in visual recognition is specific to faces and extends to problems with unfamiliar faces. RP obtained a low score (31/54) on the Benton-Van Allen face test (Benton \& Van Allen, 1968) and on the Warrington test (32/50; Warrington, 1984). Object recognition is unimpaired: RP showed good performance on the Boston naming test (56/60) as well as on the Snodgrass and Vanderwart (1980) picture naming test (115/120).

$\mathrm{RP}$ also failed to recognize well-known stimuli cartoon characters $(2 / 26)$ but four times correctly identified the animal on which the cartoon figure was based (for example, pig head for Miss Piggy) (Moscovitch et al., 1997).

\section{EXPERIMENT 1: NORMALLY ORIENTED AND INVERTED FACES}

RP's performance on matching upright stimuli was compared with his performance with inverted stimuli (presented upside down). The experiment consisted of a whole stimuli matching task and a whole-to-part matching task. Whereas in the whole stimuli matching task a strategy of looking at the whole stimulus seems most efficient, presenting a whole-to-part task encourages a parts-based strategy.

The tasks were designed such that the level of categorization is the same with face and object stimuli; matching exemplars of unfamiliar faces (exemplars of the category "face") and matching of unfamiliar objects of the same category (e.g., exemplars of the category "shoe"). In both tasks, the faces and objects were presented in upright as well as inverted orientation. Stimulus presentation and data acquisition were performed with a computer. Latency as well as accuracy were recorded, such that a possible speed-accuracy trade-off can be detected.

In line with earlier findings (De Gelder \& Rouw, 2000a) we expected that RP shows worse performance with normal face configuration than with stimuli in which this configuration is disturbed. In contrast, if RP's face recognition problem is accompanied by parts-based processing, little effect of configuration is expected. 
Participants. Sixteen students from Tilburg University received course credit to serve as normal controls.

Materials. The stimuli consisted of faces and pairs of shoes (Experiment 1A) and faces, face parts, houses, and house parts (Experiment 1B). Thirty-two faces (16 male) were photographed with a Canon Still Video Camera RC-560 on a Video Floppy Disc VF-50. Photographs were prepared as grayscale pictures with an image processing and production program (Aldus Photo Styler) for presentation on a monitor.

Faces of 16 ( 8 male) models were photographed in frontal view and three-quarters orientation. Sixteen models (eight male) were photographed only in three-quarters orientation to serve as distractor stimuli. Similarly, 16 pairs of shoes ( 8 male shoes) were photographed in frontal view (i.e., with the tip of the shoe pointing toward the camera) and in three-quarters orientation (tip turned in horizontal plane to the side of camera). Another 16 pairs of shoes were photographed only in three-quarters orientation.

With the same apparatus, frontal view photographs of houses were taken and subsequently computer edited to grayscale pictures. One prototypical picture of a house was selected to be used as framework. Only the roof and outer contour was left intact, the rest of the house was filled with an uniform grey colour. With the computer image-editing program, eight different houses were created by placing different sets of inner features (a door and two windows) from eight other photographe d houses in the outer contour. The features were always in an identical configuration such that house contour and location of the three inner house features were always the same. Thus, eight house stimuli differed only in which features were used as three inner features. For Experiment 1B, part stimuli were created by presenting the door/window (for the houses) and the eyes/mouth (for the faces) in isolation. The same stimulus material and a similar design was used in previous studies (De Gelder et al., 1998; De Gelder \& Rouw, 2000a).

Viewing distance was approximately $50 \mathrm{~cm}$, so that the stimuli subtended between 7 and $8^{\circ}$ of visual angle for length and width. In all experiments stimuli were presented to RP on a 12" screen of a Pentium laptop PC (Compaq Armada 4150 ), and to the normal participants on the 14" screen of an Olivetti desktop (M4 74 modulo). Stimulus presentation and response recording was piloted by Me12 software allowing for millisecond timing.

\section{Experiment 1A: Wholes matching task}

Design. Pictures of faces and pairs of shoes served as stimuli (see Appendices II and III for examples). Eight male and eight female faces and eight male and eight female pairs of shoes were combined with the three-quarters view of 
that same face/shoe and a different face/shoe. The 16 face and 16 shoe stimulus combinations were presented both in upright and in inverted orientation. Presentation was blocked by the two within-subject factors: stimulus class (face vs. shoe) and stimulus orientation (upright vs. inverted), with random trial presentation within a block. The experiment (four blocks) was presented twice in opposite block order to normal controls. Although the effects were significant with the controls, we feared that presenting only 32 trials per experiment to RP (since in this case there is only one participant) would create a too small range of possible errors (16 for guessing and 32 perfect score). Therefore, the experiment was presented a third time to RP.

Thus, RP saw a total of 192 (48 per condition) trials, and the normal controls saw a total of 128 (32 per condition) trials. For both normals and RP a block presented for the first time (first experiment) started with four practice trials with feedback.

Procedure. Before the start of each block, both accuracy and speed were stressed. A trial started with a warning signal, followed after $500 \mathrm{~ms}$ by presentation of the frontal pictures for $2500 \mathrm{~ms}$. After a $2500 \mathrm{~ms}$ delay (during which the screen was black), the three-quarters view target and three-quarters view distractor were presented. RP and the control participants were instructed to choose as fast as possible whether the left or right face/shoes was the same as the one they had seen previously, and to indicate their choice by pressing the corresponding key on the response box in front of them. Probes disappeared at key press. After $600 \mathrm{~ms}$ the next trial started.

Results. Latencies more than 2.5 times standard deviation from the mean RT were treated as outliers for both RP (3.1\% of data) and normal controls (maximally 6 per person, which is $4.7 \%$ ). A maximum response time of $3 \mathrm{~s}$ was set for normal controls.

Controls performed much better with upright than inverted faces, both in accuracy, $F(1,15)=66.19, p<.001$, and latency, $F(1,15)=21.6, p<.001$. Interestingly, they also showed faster performance with upright than inverted shoes, $F(1,15)=7.15, p<.05$. As Table 1 shows, RP shows a pattern of impaired upright face matching as compared with inverted face matching in accuracy though this effect is not-significant (32/48 vs. 37/48), $\chi^{2}(1)=1.29, p=$ .26 , and further RP showed a non-significant face inversion superiority in latency (3431 ms vs. $3377 \mathrm{~ms}), t(1,67)=0.09, p=.93$. Similarly, inverted shoes were matched faster $(2220 \mathrm{~ms}$ vs. $1727 \mathrm{~ms}), t(1,85)=1.36, p=.18$, and slightly better (44/48 vs. $43 / 48$ correct) than inverted shoes, but again these effects did not reach significance. 
TABLE 1

Faces and shoes matching: Upright and inverted

\begin{tabular}{lll}
\hline Stimuli & $\begin{array}{c}\text { Percentage } \\
\text { (number) correct }\end{array}$ & $\begin{array}{c}\text { Mean } R T \\
(\mathrm{~ms})\end{array}$ \\
\hline RP & & \\
Faces & & \\
$\quad$ Upright & $67 \%(32 / 48)$ & 3431 \\
Inverted & $77 \%(37 / 48)$ & 3377 \\
Shoes & & \\
Upright & $92 \%(44 / 48)$ & 2220 \\
Inverted & $90 \%(43 / 48)$ & 1727 \\
Controls & & \\
Faces & & 834 \\
Upright & $95 \%$ & $959^{* *}$ \\
Inverted & $81 \% * *$ & 729 \\
Shoes & & $773^{* *}$ \\
$\quad$ Upright & $93 \%$ & \\
Inverted & $93 \%$ & \\
\hline
\end{tabular}

Comparisons significant at $p<.05$ are indicated with $* *$.

\section{Experiment 1B: Wholes to part matching task}

Design. Stimuli were the faces, face parts, houses (see Appendix IV for an example), and house parts as described previously. Each of the eight faces was combined with two eye probes, as well as with two mouth probes. One probe was the same pair of eyes or mouth and the other part probe was pair of eyes or mouth from another face. Similarly, the houses were combined with the same attic window or door, and another attic window or door. These 16 face and 16 house stimuli combinations were presented both in upright and in inverted orientation. Thus there were, as in Experiment 1A, two within-subject factors: Stimulus class (face vs. house) and stimulus orientation (upright vs. inverted). Presentation was blocked by stimulus class and orientation, with random trial presentation within a block. Further details on the design were as in Experiment 1A.

Procedure. In this task we wished to encourage parts-based matching rather than whole-based matching. Therefore, we presented RP with a whole-to-parts matching task. Further, in the previous experiment we presented shoes as they might resemble faces in the fact that individual shoes can be distinguished on the basis of general shape information. In the next experiment, we presented object stimuli with clearly discernable and identifiable parts. Therefore, we used the houses, as described earlier, that have a similar house outline and only differ in two features: attic window and door. 
Whole stimuli were pictures of faces and houses (Appendix IV), whereas part stimuli were the eyes or mouth presented in isolation without the face context, or the door or upper window without the house context (see also description of materials).

In Experiment 1A, a frontal view target was followed by three-quarters view probes, as rotation of the face requires some global shape information and therefore would encourage whole-based rather than parts-based processing. For this same reason, Experiment $1 \mathrm{~B}$ necessarily presents both whole target and part probes in frontal view. This way, a strategy of analysing the target by selectively processing a feature (eyes and mouth) is encouraged as it would lead to optimal performance.

Again, the target appeared for $2500 \mathrm{~ms}$, followed after a $2500 \mathrm{~ms}$ delay by the two part probes. Further materials, procedure, and design were as described in Experiment I A.

Results. As in Experiment 1A, for both normal controls and RP responses longer than 2.5 standard deviations from the mean of the experiment were treated as outliers. Control participants had maximally four outliers per experiment $(3.1 \%)$ and RP had four outliers $(2.1 \%)$. In this experiment controls did not show an effect of orientation, for neither faces nor houses. Patient RP again shows an advantage with inverted stimuli. RP had a significantly better (22/48 vs. 34/48), $\chi^{2}=6.17, p<.025$, performance with inverted than upright faces. The difference in response time is non-significant (a difference of $228 \mathrm{~ms}$, standard deviations are $1274 \mathrm{~ms}$ and $1330 \mathrm{~ms}$ ) as indicated by a $t$-test, $t(1,54)=$ $0.64, p=.53$. RP further showed an interesting effect in accuracy (see Table 2) of better performance with inverted than upright presented houses $(28 / 48$ vs. $37 / 48$ correct), $\chi^{2}(1)=3.86, p<.05$. Responses were also slower with upright houses, but again this difference is non-significant, $t(1,63)=0.56, p=.57$.

\section{Discussion}

In contrast with controls, RP did not show the normal inversion inferiority effect. Instead, RP showed an inversion superiority effect; a better performance when faces are presented upside down than when they are upright. These results indicate clearly that RP does not simply rely on parts-based analysis. Indeed, if RP would completely disregard the whole stimulus and depend only on analysis of the separate face parts, similar performance would be expected on upright and inverted faces. Farah, Wilson et al. (1995) concluded that a similar finding in an inversion study with prosopagnosic patient LH indicated mandatory use of a malfunctioning "face module". This idea on the special case of faces rests on what is probably a too strong division between object and face recognition processes, as we found that prosopagnosic patient $\mathrm{LH}$ also shows inversion superiority with objects as stimuli (De Gelder \& Rouw, 2000b). With 
TABLE 2

Faces and houses matching: Upright and inverted

\begin{tabular}{llc}
\hline Stimuli & $\begin{array}{c}\text { Percentage } \\
\text { (number) correct }\end{array}$ & $\begin{array}{c}\text { Mean } R T \\
(\mathrm{~ms})\end{array}$ \\
\hline $\mathrm{RP}$ & & \\
Faces & & \\
$\quad$ Upright & $46 \%(22 / 48)$ & 2270 \\
$\quad$ Inverted & $71 \%(34 / 48)^{* *}$ & 2497 \\
Houses & & \\
Upright & $58 \%(28 / 48)$ & 3645 \\
$\quad$ Inverted & $77 \%(37 / 48)^{* *}$ & 3285 \\
Controls & & \\
Faces & & \\
Upright & $81 \%$ & 1275 \\
Inverted & $81 \%$ & 1241 \\
Houses & & 1081 \\
Upright & $86 \%$ & 1070 \\
Inverted & $83 \%$ & \\
\hline
\end{tabular}

Comparisons significant at $p<.05$ are indicated with $* *$.

this new case of prosopagnosic patient RP, we again find inversion superiority with faces as well as with objects (houses). Rather than proposing an impaired face module, we suggest that the inversion superiority effect reflects impaired use of configural processes that are involved in face recognition as well as recognition of certain classes of objects.

In our next experiments, we wished to further explore the ideas described previously on spared and impaired visual processes in prosopagnosic patient RP. There are two factors in Experiment 1 that need clarification. The first concerns the comparison between the two tasks. RP showed a pattern of inversion superiority in both tasks, but it only reached significance in the task encouraging parts-based processing rather than the task encouraging wholes-based processing. As it seems logical to expect that the influence of configuration is stronger in the task encouraging whole-based processing, this finding seems unexpected.

However, a direct comparison of performance level between the tasks is not valid given the settings and materials of Experiment 1. In this experiment, whole faces were completely different. Thus, although there are several cues in the wholes matching task (a mole, hairline), in the whole-to-part matching task the right answer can only be found in either the eyes or the mouth. Perhaps the additional information aided performance in the upright face condition, decreasing the difference between upright and inverted condition. In the next experiment, we will adapt the materials such that the two tasks are more comparable. 
The second factor concerns the comparison between upright and inverted face performance. We used this comparison to study the influence of face configuration on task performance. Although this is a widely excepted paradigm, there might be an additional effect of rotation influencing matching performance with inverted faces (for example, a face might be "mentally righted" before it can be recognized; Rock, 1974). A similar effect of rotation might influence matching performance with inverted face parts. In our next experiment, we take out this factor of rotation and present upright whole faces and face parts. The configuration of the faces is disturbed in the "scrambled faces" where face parts are put at the wrong location. Comparing a "good" and "bad" face configuration allows us to directly examine the influence of face configuration on matching performance.

\section{EXPERIMENT 2: FACE CONTEXT EFFECT WITH WHOLE OR PART PROBES}

Striking demonstrations of the influence of stimulus configuration on the perception of its constituent parts are the "word superiority effect" and the "object superiority effect". These reflect, respectively, better recognition of a letter in the context of a word (Reicher, 1969), and better recognition of a line in context of a good form (Weisstein \& Harris, 1974). Homa, Haver, and Schwartz (1976) found superior recognition performance when face parts were presented in the context of a normal whole face, as compared with the context of a scrambled (displaced features) face. These effects are not just obtained under threshold conditions, as Davidoff and Donnelly (1990) extended superiority effects to normal exposure conditions. In our previous experiment, we found that RP showed a specific disadvantage when presented with a normal face configuration as compared with a stimulus in which this configural information is assumed lost (inverted face). In our next experiment, we will try to replicate this finding by comparing a normal face configuration with a stimulus in which only this configuration has been disturbed, by displacing the face features (scrambled face). Thus, our first hypothesis is that an intact face configuration has a detrimental effect on RP's recognition performance.

Tanaka and Farah (1993) presented either a whole stimulus or a stimulus part in a recognition task, and found that face recognition, as compared with object (houses) recognition specifically profits from the presence of a whole stimulus. Indeed, these authors state that what separates face recognition from object recognition is relative little part representation with faces, as compared with objects. In a recent study, Donnelly and Davidoff (1999) examined the advantage of presenting a complete probe (wholes matching task) over a part probe (wholes-to-part matching task) with both face and house stimuli. They found a Complete Probe Advantage (CPA) with both faces and houses, and concluded that both kinds of stimuli were processed as a whole rather than in 
terms of their parts. Here we will examine the issue of whole-based vs. parts-based processing separately from the effect that an intact face configuration has on recognition performance.

First, we study the face superiority effect, which is the advantage (better performance) provided by the presence of a good face configuration. Therefore, we present facial stimuli differing only in face parts (eyes and mouth) and compare recognition performance when stimuli present a good (normal face) vs. a bad (scrambled face) context. Normal controls are expected to show the advantage with the normal face configuration as compared with the scrambled faces, as found previously by Davidoff and Donnelly (1990). RP, on the other hand, is not expected to profit from the good face context. In Experiment 1 RP showed better recognition performance if the face configuration is made less accessible (by inverting the face). In this experiment we directly disturb the face configuration by dislocating the face features. We expect to replicate the finding that RP's performance level decreases when presented with a "good" (nominal) face configuration.

Furthermore, we presented both a whole-to-whole matching task (WW) and a whole-to-part matching task (WP). In both tasks, performance with normal face stimuli is compared with performance with scrambled face stimuli. A second question, then, is whether we will find a CPA such as reported by Donnelly and Davidoff (1999). However, as these authors state, the presence or absence of a CPA might depend on several task (e.g., presentation time) and stimulus (e.g., complexity of the stimulus) settings. We did not vary these determinants and therefore cannot draw conclusion s on what constitutes the CPA. The difference between WW and WP task performance is simply seen as an indication of relative whole-based vs. more parts-based processing, but the experiment was not set up to examine the conditions in which a CPA might appear or disappear.

Control participants and RP performed the same experiment. Again (see Experiment 1) we are interested in RP's pattern of performance as compared with the pattern of performance of normal controls, rather than a direct comparison between RP and normal controls on each condition.

Participants. Twenty students from Tilburg University received course credit for participating in the experiment. As in Experiment 1, we tested a group of control participants, to establish what effects were obtained with these materials and to replicate findings as reported in the literature.

Materials. A prototype face outline was created from a black and white (photographic quality) picture of a young Caucasian male. One of six pairs of eyes and one of six mouths were digitally inserted into the face template, either at the correct position s creating six normal faces (for an example see Appendix $\mathrm{V})$ or with the eyes located at the position of the mouth and vice versa, creating 
six "scrambled" faces (see Appendix VI). Thus, the "whole faces" stimuli differed only in eye and mouth. The six pairs of eyes and six mouths could also be presented in isolation. Each (normal, scrambled) face measured $6 \times 8 \mathrm{~cm}$. The viewing distance was $50 \mathrm{~cm}$; thus the faces subtended approximately $7 \times 9^{\circ}$ of visual angle.

Design. A two-alternative forced-choice matching task was used. In the wholes matching task (WW task) a whole face target was followed by two whole face probes, one identical and one different from the target. In the whole-to-parts matching task (WP task) presentation of the whole face was followed by that of two pair of eyes or two mouths; one same and one different to the face parts presented in the whole face target.

As described earlier, we created six different faces by placing one of six eyes and one of six mouths in a standard face framework. Each face was combined with the correct probe (same face/face part) and an incorrect probe (one of the five other faces/face parts). We presented each of these combinations of target/probes (five incorrect probes for each of the six faces), making 30 trials per condition. In 15 out of these 30 cases, the correct probe was on the left side.

We studied the effect of within-subject factor stimulus type (normal vs. scrambled face) in both the wholes matching and the whole-to-part matching task. Trials were blocked by task and stimulus type, with random trial order within a block. Each block was split in two and block order was balanced within-subject. The block started with four practice trials, but only when a certain condition was presented for the first time.

Procedure. A trial started with an $800 \mathrm{~ms}$ warning signal. The test stimulus appeared for $1000 \mathrm{~ms}$, followed after a $1000 \mathrm{~ms}$ delay by a two-alternative forced choice of two part probes or two whole probes presented side by side. Normal participants responded by pressing the rightmost or leftmost key on a response box. The next trial started after a key press.

$\mathrm{RP}$ and normal controls were tested with the same procedure and materials, but RP indicated his choice by pressing one of two labelled keys on the keyboard, indicating "left" ("q") or "right" (")"). These keys were chosen as RP indicated them as most comfortable. All blocks started with an instruction, presented both on the computer screen and read aloud to RP by the experimenter (to ascertain that RP understood the task). As in Experiment 1 both speed and accuracy were stressed.

\section{Results}

One participant showed chance performance in two out of four conditions and was excluded from analyses. All other normal controls showed at least $67 \%$ correct per condition. Some of RP's latencies were much longer than his 
average response times. We excluded latencies more than three times the standard deviation from the mean from analysis (excluding $4 \%$ of the responses).

Repeated measure analyses showed that normal controls showed the expected face context effect: Controls recognized normal faces faster than scrambled faces in the wholes matching task, $F(1,18)=4.57, p<.05$. This effect was not present in the whole-to-part matching task, $F(1,18)=0.53$, $p=.48$. The effects in accuracy are very small (indeed, normal controls made very few errors) and an ANOVA on the effect of stimulus type (normal vs. scrambled) provides an $F$ value of less than 1 for both tasks. An indication of whole-based processing was provided by an overall advantage of wholes matching (WW) task, both in latency, $F(1,18)=25.77, p<.001$ and accuracy, $F(1,18)=30.59, p<.001$, over the whole-to-part matching (WP) task (see Table 3).

Interestingly, RP also showed significantly better performance on the WW $(50 / 60)$ than on the WP $(39 / 60)$ task, $\chi^{2}(1)=5.26, p=.02$. The answers were also faster but this effect was non-significant, $t(87)=0.7, p=.48$. As can be seen in Table 3, this pattern of more errors and longer latencies in the WP vs. WW task was found in each stimulus condition (both normal and scrambled faces). RP did show a context effect, but in contrast with normal controls RP showed no normal face configuration advantage. Instead, performance with normal faces was slightly worse, and significantly slower (separate variances $t$-test for equality of means: $t(69)=2.23, p=.05$ ) than scrambled face performance (see Table 3). Analysing the two tasks separately shows that the

TABLE 3

Context effect in a wholes matching (WW) task and whole-to-part machine (WP) task

\begin{tabular}{llc}
\hline Stimuli & $\begin{array}{c}\text { Percentage } \\
\text { (number) correct }\end{array}$ & $\begin{array}{c}\text { Mean RT } \\
(\mathrm{ms})\end{array}$ \\
\hline RP & & \\
WW & & \\
$\quad$ Normal & $80 \%(24 / 30)$ & $1676^{* * *}$ \\
$\quad$ Scrambled & $87 \%(26 / 30)$ & 1210 \\
WP & & \\
$\quad$ Normal & $63 \%(19 / 30)$ & 1824 \\
$\quad$ Scrambled & $67 \%(20 / 30)$ & 1356 \\
Controls & & \\
WW & & \\
$\quad$ Normal & $90 \%$ & $1055^{* * *}$ \\
$\quad$ Scrambled & $91 \%$ & 1219 \\
WP & & 1199 \\
$\quad$ Normal & $83 \%$ & \\
$\quad$ Scrambled & $81 \%$ & \\
\hline
\end{tabular}

Comparisons significant at $p<.05$ are indicated with **. 
context effect was just significant in the wholes matching (WW) task, $t(48)=$ 2.0, $p=.05$, but not in whole-to-part matching (WP) task.

\section{Discussion}

As in Experiment 1, RP showed a pattern of performance opposite to that of normal controls. Control participants showed the same effects as reported previously by Davidoff and Donnelly (1990) and Donnelly and Davidoff (1999), better recognition with normal than with scrambled face context. RP, on the other hand, showed a paradoxical pattern of worse performance with normal than scrambled faces. This worse performance with normal faces could not have been found if RP depends on parts-based procedures. Normal and scrambled faces only differ in the location of the features, therefore RP's differential performance on normal vs. scrambled faces indicates processing of "whole-based" or "relational" information.

In contrast with the previous experiment, in this experiment the tasks were more similar in overall difficulty as little extra information was given in the WW as compared with the WP task. This was done to make possible a better comparison between tasks. A surprising finding is that RP still shows the advantage of the wholes matching task over whole-to-part matching task, just as normals did. One possible explanation is that more information is present in wholes-matching, as both a different eye and different mouth are present in the foil distractor, while only one feature probe is present in the WP task. ${ }^{1}$ Another, a bit counterintuitive, explanation is an advantage of whole-based over parts-based processing for RP. Logically, the ability to process the whole stimulus is more important in the wholes matching task than in the whole-to-part matching task. At the moment, we cannot conclude whether RP's performance indeed is better with whole-based rather than parts-based processes. We can conclude, however, that RP shows a face disadvantage effect using whole-based rather than parts-based procedures. Normals show, as expected, that the face superiority effect is present in the wholes matching task but not in the whole-to-part task. For RP, in this experiment the face inferiority effect was significant in the wholes matching task (encouraging whole-based procedures) but not in the whole-to-part matching task. Although an alternative explanation can be given for RP's overall better performance on the wholes matching task than on the whole-to-part task, this does not explain why his normal face disadvantage would be found in a task encouraging whole-based processes rather than in a whole-to-part task encouraging parts-based procedures.

Prosopagnosic patients are expected to have a problem with "the whole face" and would instead rely on "parts-based analysis", but RP's case does not conform to this expectation. His pattern of performance suggests that the

\footnotetext{
${ }^{1}$ We thank N. Donnelly for pointing out this alternative explanation to us.
} 
presence of a normal face configuration disturbs rather than improves his performance, indicating whole-based rather than parts-based procedures. In the General Discussion we will return to these issues in relation to ideas and findings presented in face recognition literature. In the next experiment we will try to find further support for RP's intact "whole face encoding". Furthermore, we will examine what is included in the "whole face".

\section{EXPERIMENT 3: ENCODING THE WHOLE FACE}

In this experiment, we studied RP's "whole face processing" in a new and different manner by examining whether both external and internal facial features are included. We designed a task in which only the inner face features are to be compared, whereas hair and hairline should be ignored. Thus, the relative importance of the whole face as compared with some inner feature registration can be contrasted. If RP relies on analysis of inner features, differences in hair should not disturb his matching performance. However, if RP encodes "the whole", and this whole picture includes both the internal and external features, hair and hairline will influence his recognition of inner face features.

Materials. Black and white pictures of photographic quality from twelve faces (six male and six female) served as the basis. These were the same pictures used to create the stimuli of Experiment 1. Each face was paired with a same-sex other face that differed only minimally in easy cues such as hairstyle and overall face shape. With a photo-editing computer program (Adobe Photoshop), hair of the two faces in a pair was switched, thus creating two "new" faces from each face pair. The resulting sets of four faces each resulted in a total of 24 face stimuli. As can be seen in Appendix VII, the "swapped hair" faces were carefully edited to look like natural faces.

Design. There were six sets of four faces (two original faces and two "swapped hair" faces) each. In a trial, each face was combined with each of the four faces in its set, creating 16 trials per set and 96 trials in the whole experiment. A target face could be followed by itself, the other (paired) face, the same face but with different hair, or a different face with the same hair. Thus there were two within-subject factors, answer category ("same" or "different" face) and hair congruency ("congruent" or "incongruent" with the correct answer). The four combinations per target face were presented for each of the 24 faces, therefore there were 24 trials in each condition. The total of trials was divided in two blocks: Participants first saw a block with male faces, and after a short break a second block with female faces. Within each block, trials were randomized. 
Procedure. Participants received an explicit instruction to ignore the hair and respond to the inner face only. Two faces, presented in sequence, were compared. Two keys of a response box (keys on the keyboard for RP as in the previous experiments) were labelled "same" and "different". A trial consisted of $1 \mathrm{~s}$ presentation of a face, followed, after a $1 \mathrm{~s}$ delay, by the face probe. The second face disappeared after key-press. After $800 \mathrm{~ms}$, the next trial started.

Several measures were taken to ascertain that the instructions were understood by RP. Instructions were read aloud, and RP was encouraged to ask questions. It was stressed that only the face itself should be compared. Without mentioning that the hair had been swapped, it was said that hair was unimportant in reaching the right answer and should be ignored. Furthermore, both for $\mathrm{RP}$ and the control participants the experiment started with 16 practice trials (4 from each condition) with feedback.

Participants were seated approximately $50 \mathrm{~cm}$ from the computer screen (size of the faces subtended a visual angle of $5.1^{\circ}$ horizontal by $6.3^{\circ}$ vertical) and responded by pressing one of two keys of a response box, labelled "same" and "different". As in the previous experiments, instructions encouraged to provide an answer as fast as possible, although it was stressed that at all times participants should try to find the correct answer.

\section{Results}

Normal controls showed an interfering effect of hair and hairline, when it was incongruent with the right answer, in latency, $F(1,19)=79.34, p<.001$, as well as in accuracy, $F(1,19)=38.10, p<.001$. The interference effect is particularly manifest in "same" response condition (see Table 4): If the whole face probe is the same, mean percentage correct is $96 \%$, but this percentage decreases to $76 \%$ correct if the hair is different, $F(1,19)=40.16, p<.001$. The effect of incongruent hair was also measured in longer response times, both in "same", $F(1,19)=$ $39.84, p<.001$, and in "different", $F(1,19)=4.5, p<.05$, trials.

As with the normal controls, incongruent hair disturbed RP's performance. Overall, this effect is significant in accuracy, $\chi^{2}(1)=9.50, p=.002$, but not in latency, $t(64)=1.44, p=.15$. As can be seen in Table 4, "same" responses were fast $(1457 \mathrm{~ms})$ and had high accuracy (96\% correct) if the whole face was same. A difference was found between this same face/same hair condition and the condition of same face/different hair, as the latter condition showed non-significant longer response times $(2011 \mathrm{~ms}), t(34)=1.88, p=.07$, and more errors ( $54 \%$ correct), $\chi^{2}(1)=11.11, p<.001$. A disturbing effect of hair was observed, but was not significant, $\chi^{2}(1)=1.42, p=.23$, in the "different" trials: Congruent hair gives quite good performance (71\% with 1816 ms mean response time), whereas a different face with same hair results in chance performance (54\% with $2261 \mathrm{~ms}$ mean response time). 
TABLE 4

Interference from incongruent hair

\begin{tabular}{llc}
\hline Stimuli & $\begin{array}{c}\text { Percentage } \\
\text { (number) correct }\end{array}$ & $\begin{array}{c}\text { Mean } R T \\
(\mathrm{~ms})\end{array}$ \\
\hline $\mathrm{RP} \quad$ & \\
$\quad$ Same face & & \\
$\quad$ Same hair & $96 \%(23 / 24)$ & 1457 \\
$\quad$ Different hair & $54 \%(13 / 24)^{* *}$ & 2011 \\
Different face & & \\
$\quad$ Different hair & $71 \%(17 / 24)$ & 1816 \\
$\quad$ Same hair & $54 \%(13 / 24)$ & 2261 \\
Controls & & \\
Same face & & 877 \\
$\quad$ Same hair & $96 \%$ & $1044^{* *}$ \\
$\quad$ Different hair & $76 \% * *$ & 922 \\
Different face & & $958^{* *}$ \\
$\quad$ Different hair & $94 \%$ & \\
$\quad$ Same hair & $92 \%$ & \\
\hline
\end{tabular}

Comparisons significant at $p<.05$ are indicated with $* *$.

\section{Discussion}

Our position that RP still encodes "the whole" stimulus rather than relying completely on "face parts analysis" is supported again with this different paradigm. The findings furthermore indicate that both internal and external face features are included in this "whole face processing" by RP. An incongruent external feature does disturb his performance, even when explicitly instructed to match internal features only.

One might argue that RP performs worse with incongruent external features because he did not follow instructions and based his judgements on hair instead of on the inner face features. However, this explanation is highly unlikely. First, we provided extensive instructions and practice trials to ensure that RP would be looking at the inner features of the face. Second, if RP judged the hair instead of the face, RP's performance would approach zero percent correct in incongruent trials (judging the hair and therefore providing the wrong answer for the face). This effect was not found: In those cases where inner and outer features provided conflicting answer categories RP's performance dropped to chance but did not approach zero. This chance performance was in sharp contrast with his quite good performance if external and inner features had congruent answer categories. Another alternative explanation might be that RP processes features separately, but cannot focus on (or attend to) the inner features due to a dominating role of the hair (e.g., "more" or "easier" information). However, judging outer features would again lead to a $0 \%$ rather than a $50 \%$ score on incongruent trials. We conclude that RP's patterns of results show interference from hair, rather than judgement based on hair. An 
explanation based on a problem in directing attention is also not plausible given RP's further pattern of performance, particularly his good performance on tasks examining low-level visual processes (see Appendix I). Finally, note that in this as in the previous experiments RP is compared with himself rather than directly with normal controls. All alternative explanations on possible differences between RP and controls based on RP's generally worse performance cannot explain RP's pattern of performance: A clear advantage of "congruent hair" trials over "incongruent hair" trials.

\section{GENERAL DISCUSSION}

In Experiment $1 \mathrm{RP}$ showed, in contrast with normal controls, worse performance with upright faces than with faces presented in inverted orientation. Furthermore, "this inversion superiority effect" was observed not only with faces but also with object stimuli, as found previously with agnosic patient AD (De Gelder et al., 1998) and prosopagnosic patient LH (De Gelder \& Rouw, 2000b). RP's impaired performance when presented with a normal face configuration was repeated and extended in Experiment 2. RP showed inferior performance finding subtle differences between faces in a good (normal) face context as compared with a disturbed (scrambled) face configuration. Normal controls, however, did show superior performance with the good face context. This contradicts the older notion (e.g., Levine \& Calvanio, 1989) that impaired face processing is accompanied by dependence on parts-based analysis. Experiment 3 confirmed this notion and furthermore showed that both internal and external features are included in this "whole face" representation. In a task of matching inner face features, an incongruent external feature (prompting a different answer than the inner features) greatly disturbs RP's performance.

\section{Face configuration vs. whole-based processing}

The results from these three experiments converge in showing that RP does still process the whole face. But at the same time the presence of a face configuration disturbs rather than improves his performance. This seems contradictory, as in the past the two notions "whole-based processing" and "face configuration" have often been looked upon as closely connected or even as two ways to describe the same intact face mechanism (Tanaka \& Farah, 1993). Therefore, the main conclusion from these findings with prosopagnosic patient RP is that a strict division between "whole-based" face recognition processes on the one hand, and "parts-based" object recognition processes on the other hand does not provide a correct picture.

The distinction between "whole-based" information vs. "face configuration" information described earlier calls to mind Carey and Diamond's description of "first order relational information" vs. "second order relational 
information" (as described in the introduction to this paper). First order relational information specifies the relations between the stimulus parts and is contrasted with information of the isolated face parts, and is assumed sufficient to recognize a stimulus category (e.g., "a car" or "a face"). Recognition of individual faces, however, depends also on second-order relational information (specifying an individual by describing how it relates to the shared configuration of all faces). To the present data, this description of two different kinds of "configural" information related to face recognition is very valuable. Indeed, patient RP provides for the first time a prosopagnosic case description that clearly supports this distinction between two kinds of information. RP's performance suffers from the presence of second order relational information: Worse performance with normal than inverted (Experiment 1) or scrambled (Experiment 2) faces. Simultaneously, RP shows no disturbance of first order relational information (RP does not depend on parts-based processing). The important difference between the previous (Carey and Diamond) model and our findings lies in the fact that RP's "whole-based" processing is found in tasks at the level of individual face matching, rather than face detection or categorization. Thus, also for face recognition (identification), relative dependence on the whole face or face parts, should be examined separately from the influence of an intact vs. impaired face configuration.

We studied the degree of parts-based processing on a task of individual face matching as we had strong indications that the processes involved in the two tasks, face detection or categorization vs. individual face recognition, are not equal (Schweich \& Bruyer, 1993). For example, Ellis (1986) suggested that face decision, but not face recognition, is based upon an automatic analysis. Furthermore, in an earlier study we contrasted recognition performance of two prosopagnosic patients RP and AV (De Gelder \& Rouw, 2000a). Whereas patient RP showed worse performance with a normal face configuration, patient AV showed no effect of face configuration (equal performance with upright and inverted faces) and seemed to depend on parts-based processes. In contrast, both patients were excellent on a face categorization and face detection task, even in a speeded face detection task that did not allow a featural analysis. These findings suggest that the functioning of "whole-based" vs. "parts-based" processes should be examined separately in the case of face decision and the case of face identification.

\section{$\mathrm{RP}^{\prime}$ s impaired face recognition}

Our matching experiments were designed such that good matching performance would depend on the ability to find subtle differences between individual faces. Indeed, RP showed a specific disadvantage with a "good face configuration", suggesting that the configural processes involved in this discrimination were impaired. The finding of an inversion superiority effect with 
objects indicated that these processes are also involved in recognition of certain classes of objects. So far, we have given little theoretical consideration of what representation or processes underlie the processing of the "good face configuration" of unfamiliar faces. We did not test one model of face recognition against another. Whether faces depend on configural relations as compared with a face norm (Rhodes, Brennan, \& Carey, 1987), or are stored as exemplars in "face space" (Valentine, 1991), or are individuated relative to their "prototype", or shared configuration (Diamond \& Carey, 1986), does not make a difference for the conclusions drawn from our results. The results do indicate, however, that RP's worse performance with a good face configuration is related to making subtle within-category comparisons, rather than to "face-specific" processes.

Furthermore, the within-category comparison rather than the expertise we hold for faces seems an important factor in RP's deviant pattern of performance. An explanation based merely on the expertise we hold for faces does not provide an explanation for our findings, as the inversion superiority effect was also found with objects. These stimuli were new to RP and he had no specific expertise for shoes or houses.

It is interesting to contrast our findings with those of Davidoff and Landis (1990), who found no difference between prosopagnosic's performance on normal vs. scrambled faces. The authors concluded that the patients had a general (not face-specific) impairment in forming integrated temporary representations. These temporary representations were contrasted with representations formed "through object specific routines"; thereby forming integrated higher level representations for objects (or faces), but not for scrambled objects. As Davidoff and Donnelly (1990) noted, the difference between a scrambled face and a normal face is that only the latter can use this "higher order" information. In contrast with the patients studied by Davidoff and Landis, RP's problem does not lie in temporary representations, which would mean a similar problem with faces, objects, and non-objects (scrambled or inverted stimuli). RP, however, has a clear disadvantage with stimuli containing the "higher order" information of face class, and showed better performance with non-objects than with faces or objects. Another difference between our study and that of Davidoff and Landis is that as we separated the influence of "face class" from the relative dependence on whole- vs. parts-based processing, we did not expect a wholes matching advantage for faces only. We have made clear that RP's problem with face recognition is not dependence on parts-based processes. A next question is then of course what underlies RP's impaired face processing.

It is important to note that in this case prosopagnosia is not only a problem in familiar face recognition ("Face Recognition Units" in the model of Bruce \& Young, 1986). RP is also significantly impaired in unfamiliar face matching. We studied what visual processes underlie this deficiency and found that RP 
has a problem with those processes involved in finding subtle differences between individual unknown faces. Though it seems obvious that his problem with unfamiliar faces is related to his problem in storing and processing of familiar faces, this study does not examine all processes involved in familiar face recognition in RP.

One possibility is that besides from his deviant pattern of performance in matching faces, there is a problem in the storage of familiar faces. Another possibility is that RP has an additional visual problem, which is specifically disturbing for recognition of faces as compared with other objects. Such additional problem could for example be a difficulty in surface recognition or curved lines (Kosslyn, Hamilton, \& Bernstein, 1995). Finally, an intriguing idea is that RP's specific problem with faces results from the combination of both factors described in this study. RP has impaired ability to make subtle comparisons within a stimulus class. Normal participants are aided by the fact that this is a well-known category and depend on their knowledge of what individuals in this class roughly look like. RP, however, has an impairment in using this knowledge. Furthermore, he still processes these stimuli in a whole-based manner, just as normals do. Therefore, his normal use of the face or object configuration is impaired, while simultaneously the alternative route of analysing parts is blocked. This interaction of two factors might lead to specifically bad performance. For now, the exact interaction between these processes remains speculation.

What the results do show, in conclusion, is that severely impaired face recognition does not mean dependence on parts-based analysis. RP can and does still processes the whole face. Vice versa, intact encoding of the whole stimulus does not mean intact face expertise: Clearly RP is impaired in face recognition. We propose that at the level of face identification a distinction should be made between whole-based vs. parts-based processing on the one hand, and processes related to stored information on the face (or object) class on the other.

\section{REFERENCES}

Benton, A.L., \& Van Allen, M.W. (1968). Impairment in facial recognition in patients with cerebral disease. Cortex, 4, 344-358.

Biederman, I., \& Kalocsai, P. (1997). Neurocomputationa 1 bases of object and face recognition. Philosophical Transactions of the Royal Society London: Biological Sciences, 352, 1203-1219.

Bodamer, J. (1947). Die prosop-Agnosie. Archive für Psychiatrie und Nervenkrankheite, 179, $6-53$.

Bornstein, B. (1963). Prosopagnosia. In L. Halpern (Ed.), Problems of dynamic neurology. Jerusalem, Israel: Hadassah Medical School.

Bornstein, B., Sroka, M., \& Munitz, H. (1969). Prosopagnosi a with animal face agnosia. Cortex, $5,164-169$.

Bruce, V., \& Young, A.W. (1986). Understanding face recognition. British Journal of Psychology, 77, 305-327. 
Bruyer, R., Laterre, C., Seron, X., Feyereisen, P., Strypstein, E., Pierrard, E., \& Rectem, D. (1983). A case of prosopagnosi a with some preserved covert remembrance of familiar faces. Brain and Cognition, 2, 257-284.

Carey, S., \& Diamond, R. (1994). Are faces perceived as configuration s more by adults than by children? Visual Cognition, 1, 313-348.

Damasio, A.R., Damasio, H., \& Van Hoesen, G.W. (1982). Prosopagnosia: Anatomic basis and behavioral mechanisms. Neurology, 32, 331-341.

Davidoff, J., \& Donnelly, N. (1990). Object superiorit y effects: Complete versu s part probes. Acta Psychologica, 73, 225-243.

Davidoff, J., \& Landis, T. (1990). Recognition of unfamiliar faces. Neuropsychologia, 28, 1141-1161.

De Gelder, B., Bachoud-Levi, A.C., \& Degos, J.D. (1998). Inversion superiorit y in visual agnosia may be common to a variety of orientation polarised objects besides faces. Vision Research, 38, 2855-2861.

De Gelder, B., \& Kanwisher, N. (1999). Absence of a fusiform face area in a prosopagnosi c patient. NeuroImage, 9(6), S604.

De Gelder, B., \& Rouw, R. (2000a). Configural face processes in acquired and developmental prosopagnosia: Evidence for two separate face systems? NeuroReport, 11(14), 3145-3150.

De Gelder, B., \& Rouw, R. (2000b). Paradoxical inversion effect for faces and objects in prosopagnosia. Neuropsychologia, 38, 1271-1279.

Diamond, R., \& Carey, S. (1986). Why faces are and are not special: An effect of expertise. Journal of Experimental Psychology, 115, 107-117.

Donnelly, N., \& Davidoff, J. (1999). The mental representation s of faces and houses: Issues concerning parts and wholes. Visual Cognition, 6, 319-343.

Ellis, H.D. (1986). Introduction: Processes underlying face recognition. In R. Bruyer (Ed.), The neuropsycholog y offace perception and facial expression (pp. 1-27). Hillsdale, NJ: Lawrence Erlbaum Associates Inc.

Farah, M.J., Levinson, K.L., \& Klein, K. (1995). Face perception and within-categor y discrimination in prosopagnosia. Neuropsychologia, 33, 661-675.

Farah, M., Wilson, K., Drain, H., \& Tanaka, J. (1995). The inverted face inversion effect in prosopagnosia: Evidence for mandatory, face-specific perceptual mechanisms. Vision Research, 35(14), 2089-2093.

Farah, M.J., Wilson, K.D., Drain, M., \& Tanaka, J.N. (1998). What is "special” about face perception? Psychological Review, 105, 482-498.

Gauthier, I., Skudlarski, P., Gore, J.C., \& Anderson, A.W. (2000). Expertise for cars and birds recruits brain areas involved in face recognition. Nature Neuroscience, 3(2), 191-197.

Gauthier, I., \& Tarr, M.J. (1997). Becoming a "Greeble" expert: Exploring mechanism s for face recognition. Vision Research, 37(12), 1673-1682.

Homa, D.B., Haver, B., \& Schwartz, T. (1976). Perceptibility of schematic face stimuli: Evidence for a perceptual gestalt. Memory and Cognition, 4, 176-285.

Kosslyn, S.M., Hamilton, S.E., \& Bernstein, J.H. (1995). The perception of curvature can be selectively disrupted in prosopagnosia . Brain and Cognition, 27(1), 36-58.

Levine, D.N., \& Calvanio, R. (1989). Prosopagnosia: A defect in visual configural processing. Brain and Cognition, 10, 149-170.

McNeil, J., \& Warrington, E. (1993). Prosopagnosia: A face-specifi c disorder. Quarterly Journal of Experimental Psychology: Human Experimental Psychology, 46A(1), 1-10.

Moscovitch, M., Winocur, G., \& Behrmann, M. (1997). What is special about face recognition: Nineteen experiments on a person with visual object agnosia and dyslexia but normal face recognition. Journal of Cognitive Neuroscience, 9, 555-604.

Reicher, B.M. (1969). Perceptual recognition as a function of meaningfulnes s of stimulus material. Journal of Experimental Psychology, 81, 275-280. 
Rhodes, G., Brennan, S., \& Carey, S. (1987). Identification and ratings of caricatures: Implications for mental representations of faces. Cognitive Psychology, 19, 473-497.

Riddoch, M. J., \& Humphreys, G.W. (1993). Birmingham object recognition battery. Hove, UK: Lawrence Erlbaum Associates Ltd.

Rock, I. (1974). The perception of disoriented figures. Scientific American, 230, 78-85.

Schweich, M., \& Bruyer, R. (1993). Heterogeneity in the cognitive manifestations of prosopagnosia: The study of a group of single cases. Cognitive Neuropsycholog y, 10(6), 529-547.

Snodgrass, J.G., \& Vanderward, M. (1980). A standardize d set of 260 pictures: Norms for name agreement, image agreement, familiarity, and visual complexity. Journal of Experimental Psychology: Human Learning and Memory, 6, 174-215.

Tanaka, J.W., \& Farah, M.J. (1993). Parts and wholes in face recognition . Quarterly Journal of Experimental Psychology, 46A, 225-245.

Valentine, T. (1991). A unified account of the effects of distinctiveness, inversion, and race in face recognition. Quarterly Journal of Experimental Psychology, 43A, 161-204.

Warrington, E.K. (1984). Recognition memory test. Windsor, UK: NFER Nelson.

Weisstein, N., \& Harris, C.S. (1974). Visual detection of line segments: An object superiority effect. Science, 186, 752-755.

Manuscript received March 2000

Revised manuscript received March 2001

\section{APPENDICES}

\section{Performance of patient RP on standardized visual processing tasks}

\section{Low level visual processes}

Birmingham Object Recognition Battery (Riddoch \& Humphreys, 1993)

$\begin{array}{lll} & R P & \text { Normal mea } \\ \text { line length (test 2) } & 24 / 30 \text { (normal) } & 26.9(1.6) \\ \text { size (test 3) } & 26 / 30 \text { (normal) } & 27.3(2.4) \\ \text { orientation (test 4) } & 23 / 30 \text { (normal) } & 24.8(2.6) \\ \text { gap (test 5) } & 37 / 40 \text { (normal) } & 35.1(4.0) \\ \text { minimal feature match (test 7) } & 22 / 25 \text { (normal) } & 23.3(2.0) \\ \text { foreshortened views (test 8) } & 23 / 25 \text { (normal) } & 21.6(2.6) \\ \text { object decision (test 10B, "hard") } & 27 / 32 \text { (normal) } & 25.4(4.7)\end{array}$

\section{Object recognition}

Boston naming test

$56 / 60$ (normal)

Snodgrass and Vanderwart picture naming (1980)

$115 / 120$ (normal)

\section{Face recognition}

Warrington

Benton

Cartoon faces

2/26 (impaired) 
II. Face stimuli of Experiment $1 \mathrm{~A}$
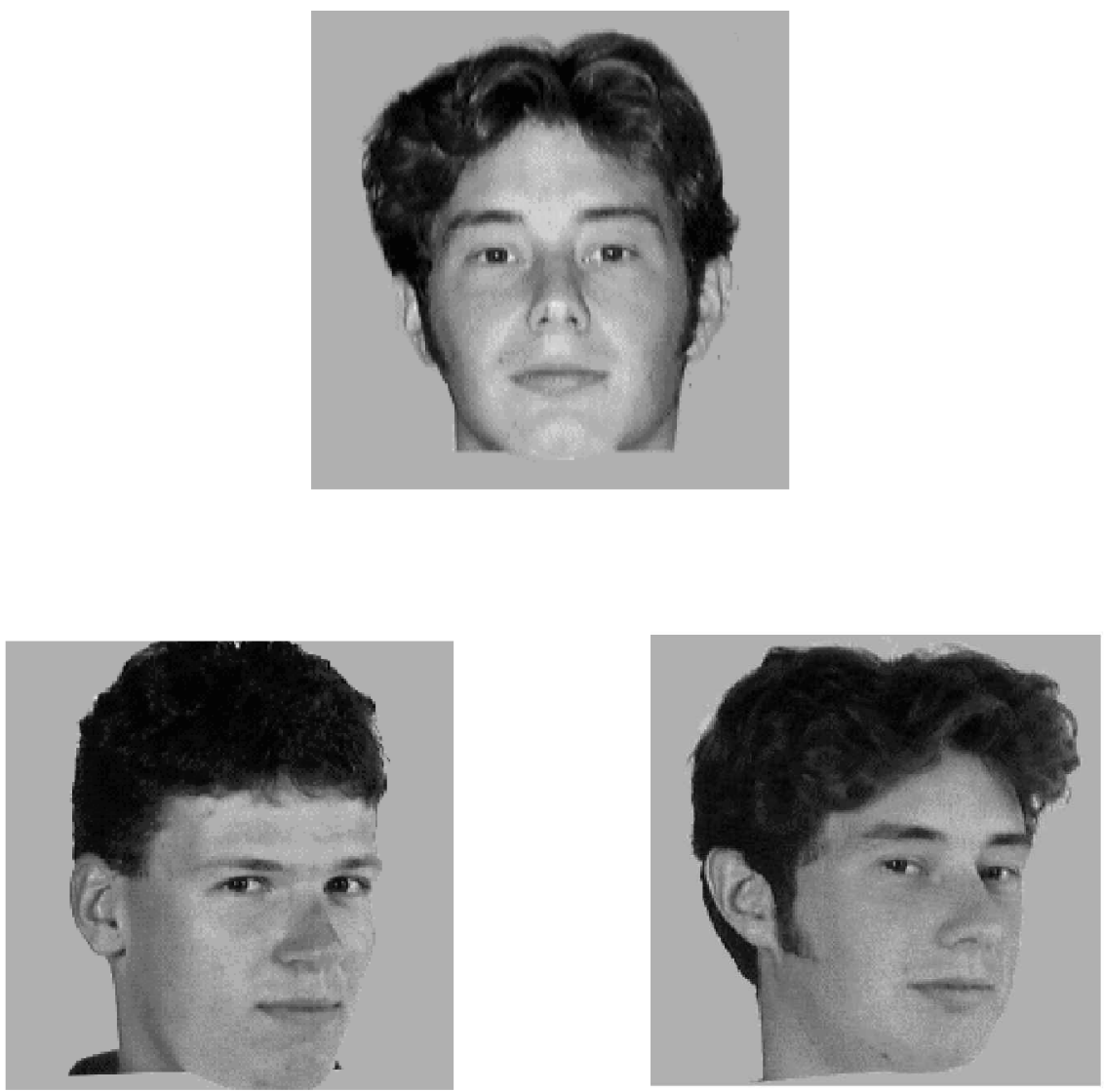
714 ROUW AND DE GELDER

III. Shoe stimuli of Experiment 1A
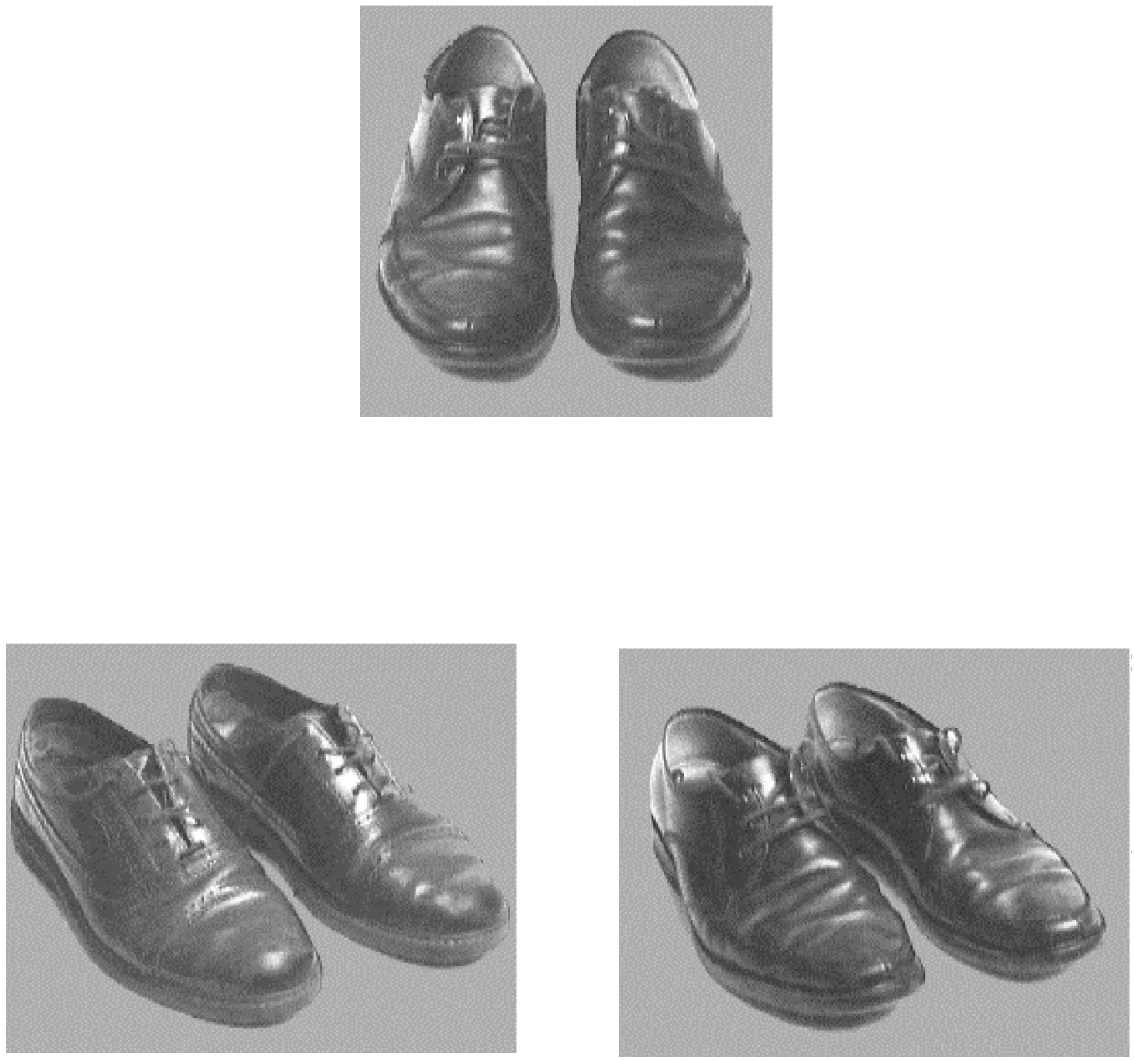
IV. House stimuli of Experiment 1B
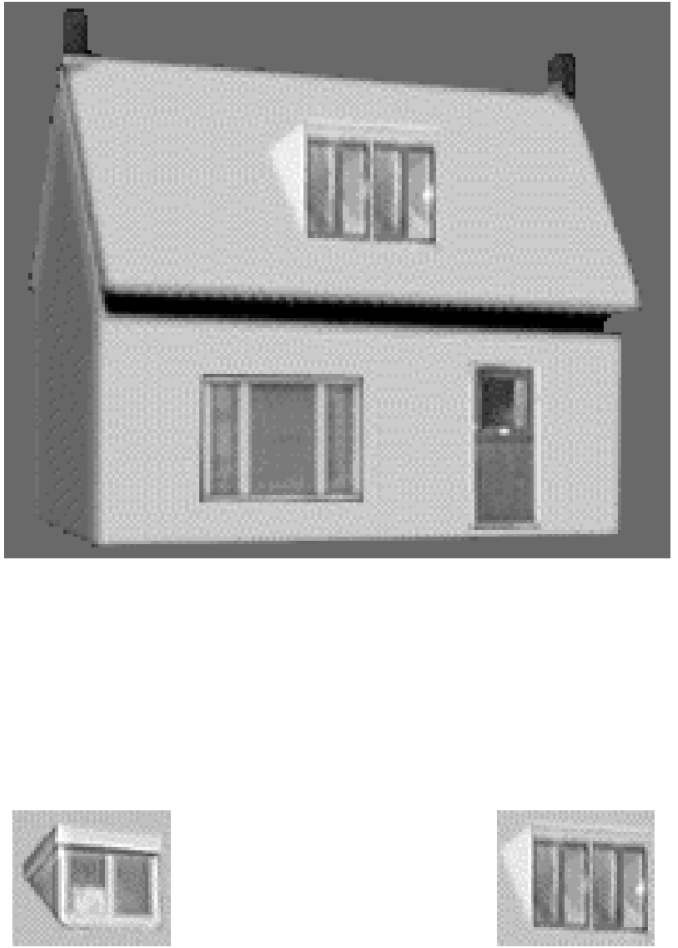
716 ROUW AND DE GELDER

V. Normal face stimuli in wholes matching task of Experiment 2
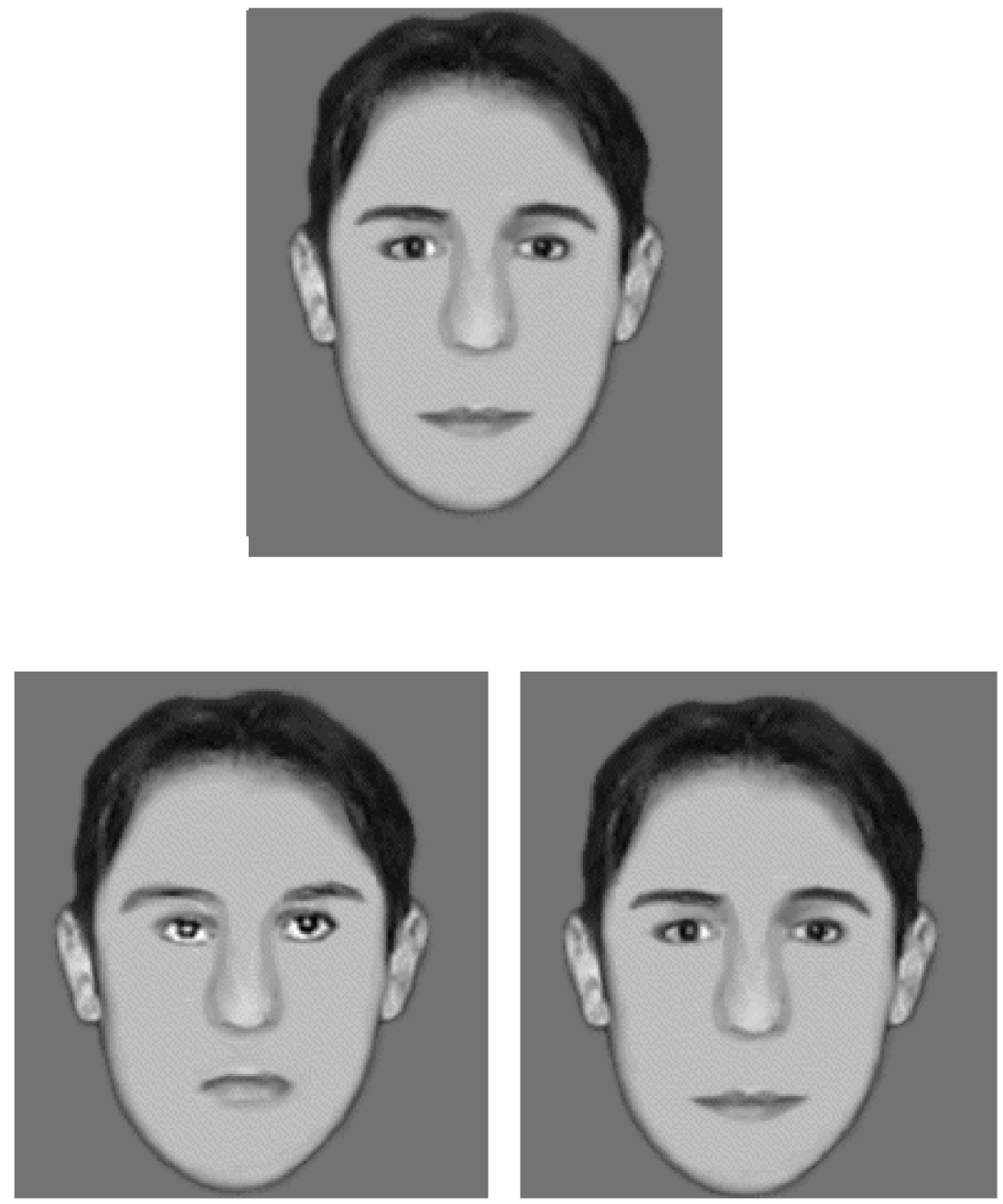
VI. Scrambled face stimuli in wholes matching task of Experiment 2
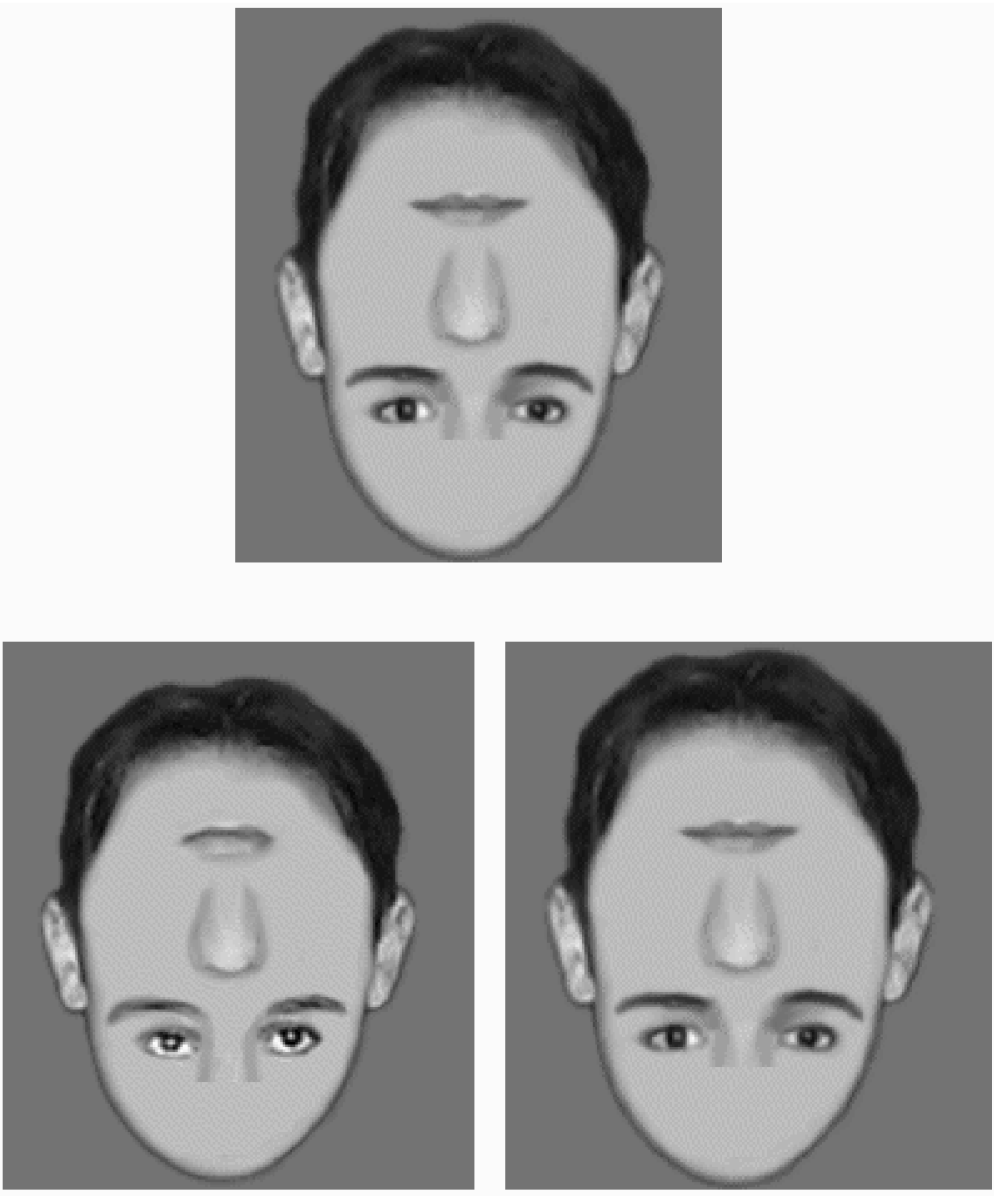
718 ROUW AND DE GELDER

VII. Example of original and changed external features faces of Experiment 3
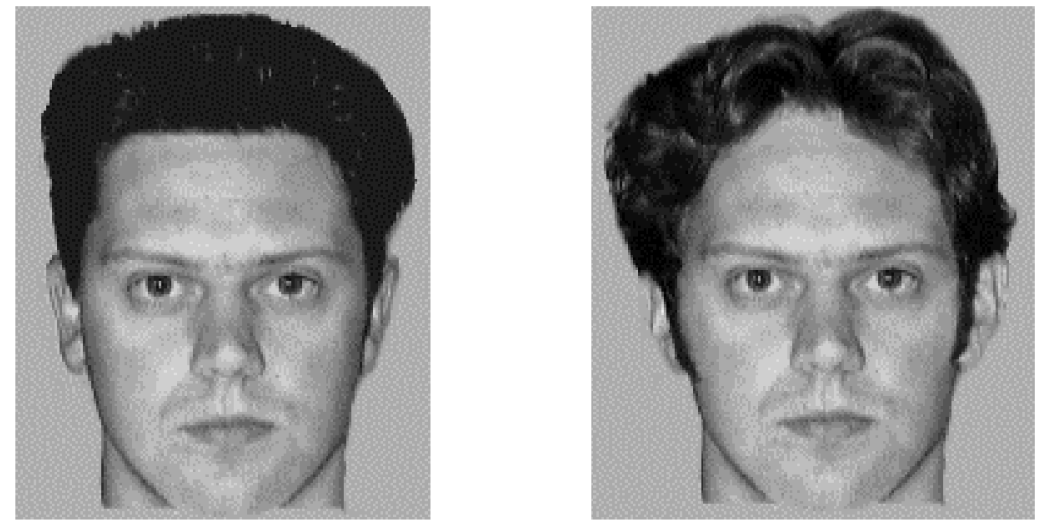